\title{
A NOVEL QRS DETECTION ALGORITHM APPLIED TO THE ANALYSIS FOR HEART RATE VARIABILITY OF PATIENTS WITH SLEEP APNEA
}

\author{
Ren-Guey Lee ${ }^{1}$, I-Chi Chou ${ }^{1}$, Chien-Chih LaI ${ }^{1}$, Ming-Hsiu LiU ${ }^{1}$, Ming-Jang ChiU ${ }^{2}$ \\ ${ }^{1}$ Institute of Computer and Communication Engineering, \\ National Taipei University of Technology, \\ ${ }^{2}$ Department of Neurology, National Taiwan University Hospital, Taipei, Taiwan
}

\begin{abstract}
Sleep-related breathing disorders can cause heart rate changes known as cyclical variation. The heart rate variation of patients with obstructive sleep apnea syndrome (OSAS) is more prominent in sleep. For this reason, to analyze heart rate variability (HRV) of patients with sleep apnea is a very important issue that can assist physicians to diagnose and give suitable treatment for patients. In this paper, a novel QRS detection algorithm is developed and applied to the analysis for HRV of patients with sleep apnea. The advantageous of the proposed algorithm is the combination of digital filtering and reverse $R$ wave detection techniques to enhance the accuracy of $R$ wave detection and easily implement into portable ECG monitoring system with light complexities of computation. The proposed algorithm is verified by simulation and experimental results.
\end{abstract}

Biomed Eng Appl Basis Comm, 2005(October); 17: 258-262.

Keywords: heart rate variability, QRS detection, sleep apnea.

\section{INTRODUCTION}

Heart rate and heart rate variability (HRV) during sleep are under the control of the autonomous nervous system. The features of sleep-related breathing disorders are repetitive cessations of respiratory flow and concomitant drops in oxygen saturation; moreover, the variation of heart rate is also obvious. Obstructive sleep apnea (OSA) is the most well-known manifestation of sleep-related breathing disorders. Sleep apnea is characterized by repetitive pauses in respiratory flow of at least 10 seconds which can occur

Received: April 15, 2005; Accepted: Aug. 22, 2005 Correspondence: Ren-Guey Lee, Professor Institute of Computer and Communication Engineering, National Taipei University of Technology, Taipei, Taiwan E-mail: evans@ntut.edu.tw up to 600 times in one night, and also affects HRV during sleep. In addition the repetitive apneas are accompanied by a pronounced increased variation in heart rate which is strong enough to support diagnosis. Hence, the characteristic pattern of bradycardia and tachycardia during sleep apnea is important information [1].

This study is aimed at $\mathrm{R}$ wave detection algorithm of electrocardiogram (ECG) signals for patients with sleep apnea and then to analyze and get the information of HRV, that can assist physicians to diagnose and give suitable treatment for patients. Besides, the telecare device for OSA patients can be developed with a light complexity of computation method for $\mathrm{R}$ wave detection. In this paper, the proposed algorithm can execute in micro-controller for this purpose.

This paper is organized as follows. In Section 2, 
the methodology of our proposed system is given. Section 3 demonstrates the results of our system. Finally, we conclude our paper in Section 4.

\section{METHOD}

Generally, the commonly used automatic ECG recognition techniques include two parts: characteristics extraction, and waveform classification and recognition [2-5]. In this paper, the QRS wave detection algorithm is used as the characteristics extraction technique. The $\mathrm{R}$ wave is the most outstanding characteristic waveform in ECG signals since it usually has the highest or lowest value in QRS complex wave. The $\mathrm{R}$ wave detection is closely related to the discrimination of normal ECG cycle and the calculation of HRV.

\section{A. R Wave Detection Algorithm}

The $\mathrm{R}$ wave detection technique used in this paper is "Modified So and Chan" $\mathrm{R}$ wave detection algorithm (MSC algorithm) [2] that combined and modified from "So and Chan" and "Tompkins" algorithms. The most advantageous of the MSC algorithm is the combination of digital filtering and reverse $\mathrm{R}$ wave detection techniques to implement into portable ECG monitoring system.

The flowchart of MSC algorithm with the ability of real-time analysis is depicted in Fig. 1. Basically it can be divided into 6 steps, namely, digital band-pass filtering, signal slope calculation, slope threshold calculation, ECG QRS wave onset detection, R wave location searching, and slope threshold update. The execution steps of the algorithm are described in detail as follows:

(1) Digital band-pass filtering: The band-pass filter can be used to reduce muscle noise, $60 \mathrm{~Hz}$ power-line interference, baseline wander, and $\mathrm{T}$ wave interference. The setting of band-pass filter is based on the frequency of ECG QRS complex wave from 5 to $15 \mathrm{~Hz}$.

a. Low-pass filter:

The transfer function of low-pass filter in bodyattached device is described by

$$
H(z)=\frac{\left(1-z^{-6}\right)^{2}}{\left(1-z^{-1}\right)^{2}}
$$

and the difference equation of low-pass filter is given by

$$
\begin{aligned}
y(n T)= & 2 y(n T-T)-y(n T-2 T)+x(n T) \\
& -2 x(n T-6 T)+x(n T-12 T)
\end{aligned}
$$

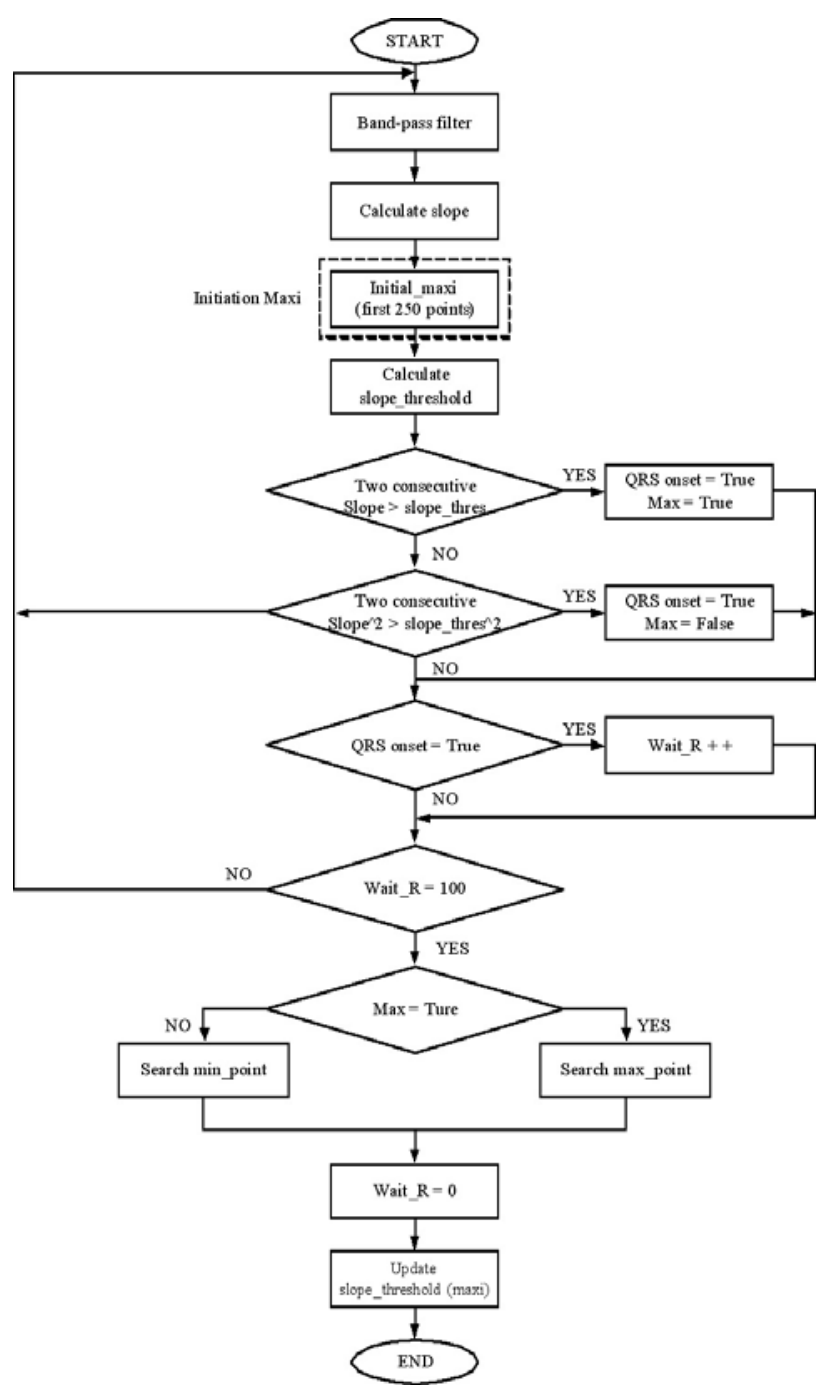

Fig 1. The flowchart of "Modified So and Chan" $R$ wave detection algorithm.

The cut-off frequency of low-pass filter is $12 \mathrm{~Hz}$. b. High-pass filter:

The transfer function of high-pass filter is described by

$$
H(z)=\frac{\left(-1+32 z^{-16}+z^{-32}\right)}{\left(1-z^{-1}\right)}
$$

and the difference equation of high-pass filter is given by

$$
\begin{aligned}
y(n T)= & 32 x(n T-16 T)-[y(n T-T) \\
& +x(n T)-x(n T-32 T)]
\end{aligned}
$$


The cut-off frequency of high-pass filter is $5 \mathrm{~Hz}$, and the gain is 32 .

(2) Signal slope calculation: The slope equation of ECG signal is described by

$$
\begin{aligned}
\text { slope }(n)= & -2 X(n-2)-X(n-1) \\
& +X(n+1)+2 X(n+2)
\end{aligned}
$$

where $X(n)$ represents the amplitude of the $\mathrm{n}$-th received discrete ECG signal.

(3) Slope threshold calculation: The slope threshold equation is described by

$$
\text { slope_thresh }=\frac{\text { thresh_param }}{16} \times \operatorname{maxi}
$$

where slope thresh represents the ratio to the maximum slope. The smaller slope threshold has the higher sensitivity to detect $\mathrm{R}$ wave but the lower immunity to noise. On the contrary, the larger slope threshold has the better immunity to noise but the lower sensitivity to $\mathrm{R}$ wave detection.

(4) ECG QRS wave onset detection: The QRS wave onset can be detected by using either of the following two conditions, slope $(n)>$ slope_thresh or slope $^{2}(n)>$ slope thresh ${ }^{2}$.

Normally the waveform of noise interference is high and sharp. That is, the slope of a single noise point usually exceeds the slope threshold and produces a false detection in the algorithm. However, for two consecutive points, if both of the slopes are larger than the slope threshold or both of the square of slopes are larger than the square of slope threshold, these two points are most probably not the noise signals and can be used the onset of QRS wave.

(5) $R$ wave location searching: According to the parameter setting in the preceding step, there can be two kinds of searching results: positive and reverse $R$ wave detection.

a. Search for the maximum value in the region: positive $\mathrm{R}$ wave

b. Search for the minimum value in the region: reverse $\mathrm{R}$ wave

(6) Slope threshold update: Every time when R wave detection is completed, maxi must be updated. The update equation is described by

$$
\begin{aligned}
& \operatorname{maxi}=\frac{\text { first_max }- \text { maxi }}{\text { filter_param }}+\text { maxi } \\
& \text { first_max }= \mid \text { height of } R \text { point } \\
&- \text { height of } Q R S \text { onset } \mid
\end{aligned}
$$

The filter parameter maxi is closely related to the sensitivity of detection to $\mathrm{R}$ waves with different amplitudes. The lower the maxi, the higher the sensitivity of detection to the amplitude difference between neighboring R-R waves. Otherwise, the sensitivity would be lower. The first max parameter represents the difference of height between QRS wave onset and apex of $\mathrm{R}$ wave. Since we have to consider the reverse $\mathrm{R}$ waves, the absolute value of first_max is taken to ensure that first max is always positive.

The searching results of $\mathrm{R}$ waves are as shown in Fig. 2(a). The ' $x$ ' , ' + ' , and ' $O$ ' in Fig. 2(a) represent the positions of QRS wave onset (positive $R$ wave), QRS wave onset (reverse $\mathrm{R}$ wave), and $\mathrm{R}$ wave respectively. Every time when an $\mathrm{R}$ wave is detected, the slope threshold will be updated by using (7) and (8). According to the $\mathrm{R}$ wave detection results, two useful data can be acquired. One is HRV and the other is $\mathrm{R}$ wave absolute location. The equation to calculate HRV is described by

$$
H R V=\left(\frac{\text { Sample_Rate }}{\text { RR_interval }}\right) \times 60
$$

The curve of HRV is shown in Fig. 2(b).

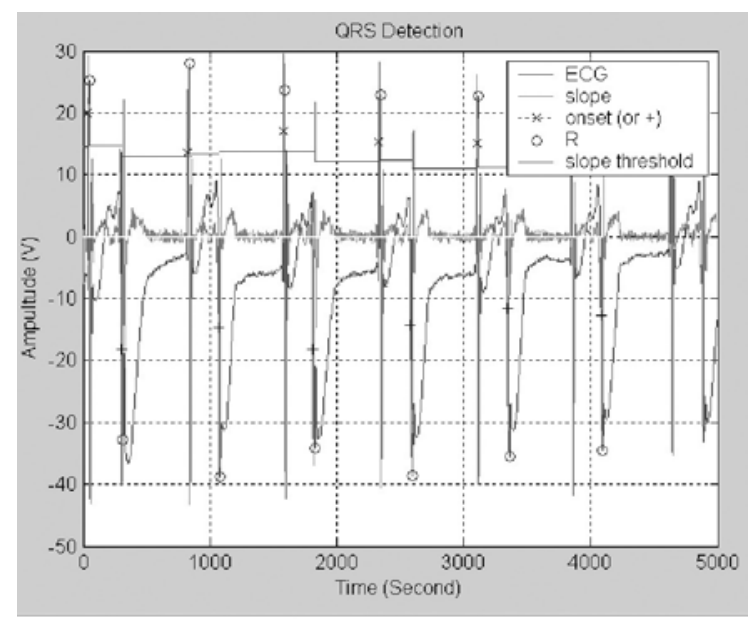

(a)

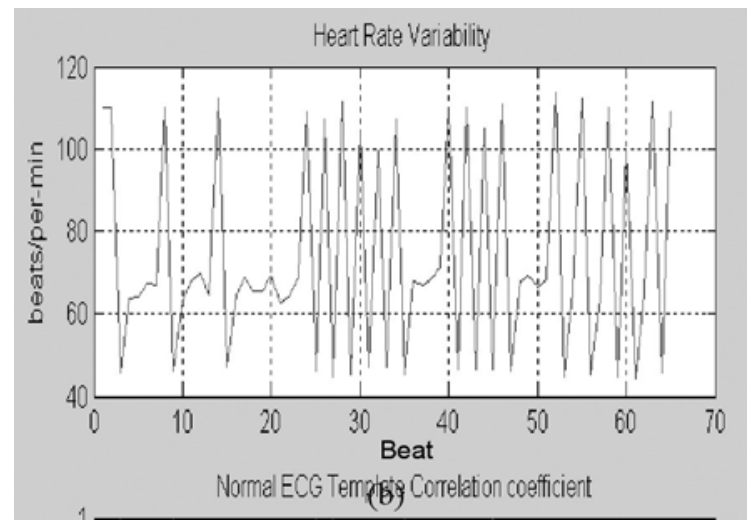

Fig 2. (a) The results of $R$ wave detection, (b) The curve of HRV of Record 119 in MIT-BIH database. 
MIT-BIH arrhythmia database has been used to verify the accuracy of MSC algorithm. We have also used 46 sets of the ECG record files in MIT-BIH database to test the accuracy of our system and the result is as shown in Table I. From Table I we can see that the average accuracy for the 46 sets of data is $95 \%$ which is acceptable. That is, our proposed algorithm can effectively detect the locations of $\mathrm{R}$-waves, and by monitoring the intervals between R-waves, and it can also effectively detect abnormal HRV conditions.

\section{RESULT}

In this section, the ECG data of patient A with OSA of National Taiwan University Hospital (NTUH)

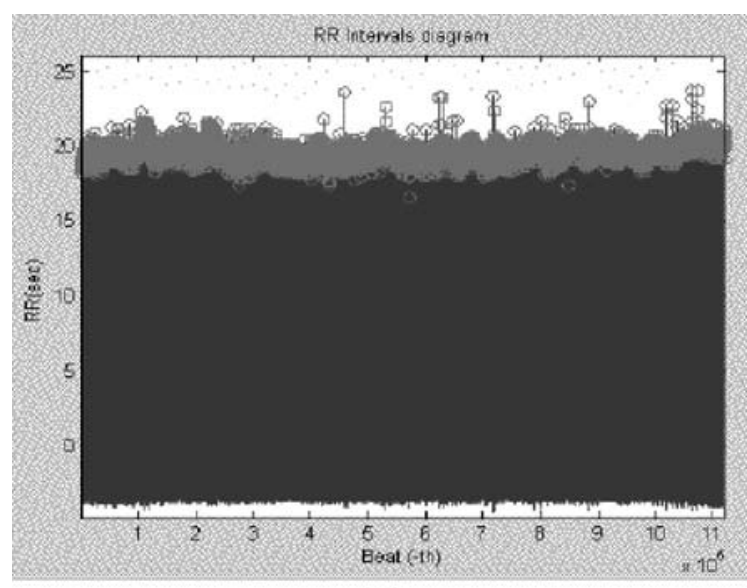

(a)

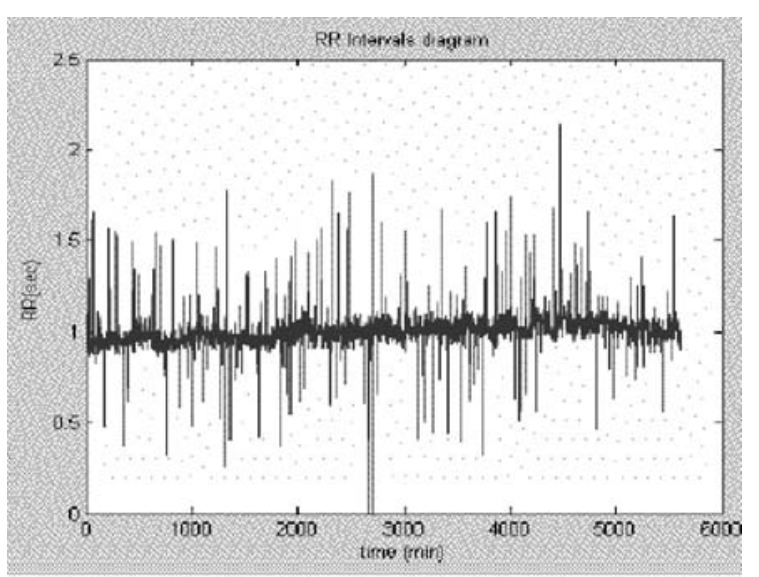

(b)

Fig 3. The waveforms of $H R V$ analysis in patient $A$ of NTUH: (a) R-R interval per beat, (b) R-R interval per minute.
Table I . Results of testing by using 46 sets of ECG record files in MIT-BIH database

\begin{tabular}{|c|c|c|c|c|}
\hline Record & Total Beats & $\mathrm{FP}^{*}$ & $\mathrm{FN}^{\dagger}$ & Accuracy ${ }^{\ddagger}$ \\
\hline 100 & 2272 & 0 & 0 & $100 \%$ \\
\hline 101 & 1870 & 6 & 1 & $100 \%$ \\
\hline 102 & 2187 & 63 & 63 & $94 \%$ \\
\hline 103 & 2083 & 0 & 1 & $100 \%$ \\
\hline 104 & 2322 & 135 & 42 & $92 \%$ \\
\hline 105 & 1959 & 170 & 783 & $51 \%$ \\
\hline 106 & 1927 & 20 & 120 & $93 \%$ \\
\hline 107 & 2135 & 39 & 41 & $96 \%$ \\
\hline 109 & 2452 & 78 & 158 & $90 \%$ \\
\hline 111 & 2123 & 6 & 7 & $99 \%$ \\
\hline 112 & 2556 & 18 & 1 & $99 \%$ \\
\hline 113 & 1794 & 0 & 0 & $100 \%$ \\
\hline 114 & 1878 & 2 & 3 & $100 \%$ \\
\hline 115 & 1953 & 0 & 0 & $100 \%$ \\
\hline 116 & 2390 & 13 & 35 & $98 \%$ \\
\hline 117 & 1537 & 4 & 2 & $100 \%$ \\
\hline 118 & 2291 & 16 & 13 & $99 \%$ \\
\hline 119 & 1987 & 5 & 5 & $99 \%$ \\
\hline 121 & 1862 & 3 & 4 & $100 \%$ \\
\hline 122 & 2476 & 1 & 1 & $100 \%$ \\
\hline 123 & 1518 & 0 & 0 & $100 \%$ \\
\hline 124 & 1605 & 14 & 28 & $97 \%$ \\
\hline 200 & 2593 & 22 & 30 & $98 \%$ \\
\hline 201 & 1933 & 173 & 240 & $79 \%$ \\
\hline 202 & 2123 & 6 & 19 & $99 \%$ \\
\hline 203 & 2839 & 284 & 425 & $75 \%$ \\
\hline 205 & 2652 & 0 & 4 & $100 \%$ \\
\hline 207 & 2072 & 210 & 470 & $67 \%$ \\
\hline 208 & 2919 & 48 & 84 & $95 \%$ \\
\hline 209 & 3008 & 9 & 5 & $100 \%$ \\
\hline 210 & 2551 & 61 & 160 & $91 \%$ \\
\hline 212 & 2749 & 2 & 1 & $100 \%$ \\
\hline 213 & 3243 & 32 & 39 & $98 \%$ \\
\hline 214 & 2261 & 26 & 26 & $98 \%$ \\
\hline 215 & 3365 & 6 & 4 & $100 \%$ \\
\hline 217 & 2210 & 14 & 12 & $99 \%$ \\
\hline 219 & 2148 & 2 & 141 & $93 \%$ \\
\hline 220 & 2048 & 0 & 0 & $100 \%$ \\
\hline 221 & 2413 & 0 & 14 & $99 \%$ \\
\hline 222 & 2486 & 49 & 46 & $96 \%$ \\
\hline 223 & 2563 & 37 & 79 & $95 \%$ \\
\hline 228 & 2034 & 82 & 101 & $91 \%$ \\
\hline 230 & 2256 & 1 & 1 & $100 \%$ \\
\hline 231 & 1571 & 0 & 2 & $100 \%$ \\
\hline 232 & 1806 & 35 & 9 & $98 \%$ \\
\hline 234 & 2752 & 0 & 1 & $100 \%$ \\
\hline \multicolumn{4}{|r|}{ Average } & $95 \%$ \\
\hline
\end{tabular}

*FP: Number of false detections when there exist no beats but detected as "beats exist"

FN: Number of false detections when there exist beats but detected as "beats does not exist"

Accuracy = 1- (FP+FN)/(Total Beats $)$ 


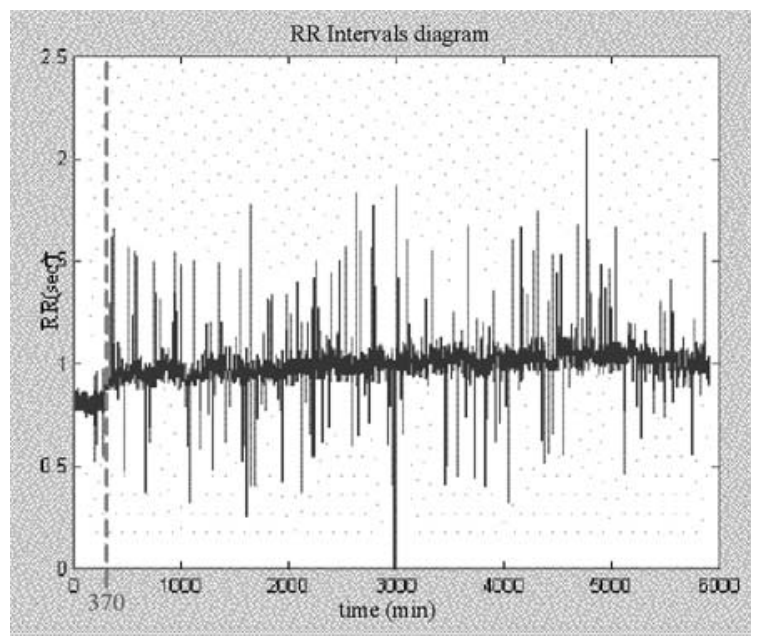

Fig 4. HRV comparison of normal and obstructive sleep apnea. (Before $370 \mathrm{~min}$. is normal; after $\mathbf{3 7 0}$ min. in patient $A$ of NTUH)

has been used to verify the feasibility of the proposed algorithm, and the practical results are presented. Fig. 3 presents respectively the HRV related waveforms of patient A. Moreover, the HRV differences of normal and OSA are shown in Fig. 4. These results confirm the feasibility of the proposed algorithm, and can support physicians to diagnose.

\section{CONCLUSION}

In this work, a novel QRS detection algorithm is proposed. Its theoretical evolution and applied results are also presented. The proposed algorithm can precisely detect $\mathrm{R}$ wave and further to analyze HRV of patients with sleep apnea. The acquired HRV information via the proposed algorithm can assist physicians to diagnose and give suitable treatment for patients with sleep apnea.

\section{REFERENCE}

1. Penzel T, Kantelhardt JW, Grote L, Peter JH, and Bunde A: Comparison of detrended fluctuation analysis and spectral analysis for heart rate variability in sleep and sleep apnea. IEEE Trans. Biomed. Eng. 2003; 50: 1143-1151.

2. Chen CC: A Portable Tele-Emergent System Supporting Electrocardiogram Discrimination. M.S. Thesis, Institute of Computer, Communication and Control, National Taipei University of Technology, Taiwan, June 2004.
3. Jiapu $\mathrm{P}$ and Tompkins WJ: A real-time QRS detection algorithm. IEEE Trans. Biomed. Eng. 1985; 32: 230-236.

4. So HH and Chan KL: Development of QRS detection method for real-time ambulatory cardiac monitor. Proceedings of the 19th Annual International Conference of the IEEE Engineering in Medicine and Biology Society 1997; 1: 289-292.

5. Tan KF, Chan KL, and Choi K: Detection of the QRS complex, $P$ wave and $T$ wave in electrocardiogram. First International Conference on Advances in Medical Signal and Information Processing Proceedings 2000; 41-47. 